\title{
Efficacy and safety of SGLT-2i in overweight/obese, non-diabetic individuals: a meta-analysis of randomized controlled trials
}

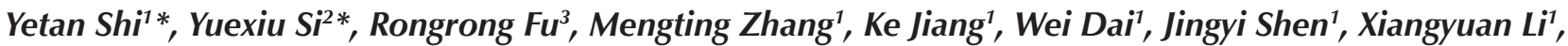 \\ Yuan Yuan ${ }^{4}$ \\ ${ }^{1}$ The Second Clinical Medical College, Zhejiang Chinese Medical University, Hangzhou, Zhejiang, China \\ ${ }^{2}$ School of Basic Medical Sciences, Zhejiang Chinese Medical University, Hangzhou, Zhejiang, China \\ ${ }^{3}$ The First Clinical Medical College, Zhejiang Chinese Medical University, Hangzhou, Zhejiang, China \\ ${ }^{4}$ Department of General Surgery, HwaMei Hospital, University of Chinese Academy of Sciences, Ningbo, Zhejiang, China \\ ${ }^{*}$ These authors contributed equally to this work.
}

\begin{abstract}
Introduction: Sodium-glucose cotransporter 2 inhibitor (SGLT-2i) has been shown to decrease blood glucose levels in type 2 diabetes mellitus (T2DM) patients and potentially yield additional benefits in weight loss. This meta-analysis aimed to investigate the efficacy and safety of giving SGLT-2i to overweight/obese, non-diabetic individuals.

Material and methods: The search was underpinned by PubMed, Cochrane Central Register of Controlled Trials, Web of Science, and Springer to identify English-language papers on randomized controlled trials (RCTs) on the use of SGLT-2i in overweight/obese, nondiabetic individuals published in and before March 2021, to study its effectiveness and safety. Results were evaluated by weighted mean difference (WMD), standardized mean difference (SMD), risk ratio (RR), and 95\% confidence interval (CI).

Results: We reviewed 13 papers and compared the SGLT-2i group with the control group (other drugs and placebo) and found that SGLT-2i reduced weight $(\mathrm{WMD}=-1.33, \mathrm{p}=0.002)$ and waist circumference $(\mathrm{WMD}=-1.94, \mathrm{p}=0.03)$ in overweight/obese, non-diabetic individuals. The use of SGLT-2i is more effective than other interventions in terms of weight loss $\geq 5 \%$ ( $R R=2.04, p<0.001)$, but not in terms of weight loss $\geq 10 \%(R R=1.83, p=0.22)$. In addition, there were no significant changes in other metabolic parameters, like fasting plasma glucose (FPG), lipids, blood pressure, etc. SGLT-2i increased the risk of infections in urinary tract $(R R=1.91, p=0.009)$ and reproductive system $(R R=4.09, \mathrm{p}<0.001)$.

Conclusions: SGLT-2i is a promising candidate to reduce weight and waist circumference to a limited extent in overweight/obese, nondiabetic individuals. Generally, it is safe and effective. However, it potentially increased the risk of urogenital infections, which cannot be ignored. (Endokrynol Pol 2022; 73 (1): 71-80)
\end{abstract}

Key words: SGLT-2i; overweight; obese; weight reduction; non-diabetic; meta-analysis

\section{Introduction}

With the rapid development of social economy, the way of people's life and diet have undergone great changes. Overweight [body mass index (BMI) $\geq 25 \mathrm{~kg} / \mathrm{m}^{2}$ ] and obesity $\left(\mathrm{BMI} \geq 30 \mathrm{~kg} / \mathrm{m}^{2}\right)$ have become a seriously public health problem [1]. Currently, approximately 1.9 billion adults around the world are overweight or obese [2]. Alarmingly, an elevated BMI is associated with increased risk of various kinds of chronic diseases, including cardiovascular disease, diabetes, cancers, etc. [3].

At present, non-surgical treatments for obesity, such as lifestyle intervention and dietary restrictions, need strict self-control of the patients and the processes are painful and arduous [4]. Hence, achieving an ideal weight or maintaining long-term weight loss process may be difficult from an individual's perspective. Obesity control guidelines recommend lifestyle interventions and medication for the overweight population [5]. So far, only four kinds of anti-obesity drugs (orlistat, phentermine/topiramate, naltrexone/bupropion, and liraglutide/semaglutide) have been approved by the US Food and Drug Administration for sustained weight management $[5,6]$. The effectiveness of orlistat, phentermine/topiramate, and naltrexone/bupropion is limited, the safety is unknown, and the use cycle is long. According to the limited data available, some glucagon-like peptide 1 receptor agonists (liraglutide/semaglutide) can lead to good weight loss and have high safety [6]. At present, more efforts are being made to seek new anti-obesity drugs with higher efficacy and more assured safety. 
Sodium-glucose cotransporter 2 inhibitor (SGLT-2i) is a class of drugs registered, according to the product characteristics, for the treatment of not only type 2 diabetes mellitus (T2DM) but also for people without carbohydrate disorders presenting heart failure and/or chronic kidney disease. Furthermore, T2DM patients treated with SGLT-2i have shown significant weight loss compared to those with placebo [7]. Therefore, some randomized controlled trials (RCTs) are designed to apply SGLT-2i to overweight/obese, non-diabetic individuals in order to reduce body weight with protection in cardiac and renal function. Some results have been obtained, but the conclusions have not been widely accepted due to the limited sample sizes. In order to enhance the credibility of the conclusions and further explore the safety and effectiveness of SGLT-2i, especially in terms of weight of the overweight/obese with non-diabetic individuals, this meta-analysis was performed.

\section{Material and methods}

\section{Literature search}

This meta-analysis was based on the Preferred Reporting Items for Systematic Reviews and Meta-Analyses guidelines [8]. Two researchers conducted a comprehensive, systematic search from the PubMed, Cochrane Central Register of Controlled Trials, Web of Science, and Springer databases. English literature documenting data was retrieved, published in March 2021 and before, on RCTs of the use of SGLT-2i in overweight/obese, non-diabetic individuals, to study the efficacy and safety of SGLT-2i in weight loss and other aspects in this population. The retrieval formula is as follows: (dapagliflozin OR canagliflozin OR empagliflozin OR ipragliflozin OR tofogliflozin OR ertugliflozin OR luseogliflozin OR bexagliflozin OR sotagliflozin OR janagliflozin OR sergliflozin OR licogliflozin OR remogliflozin OR sodium-glucose cotransporter 2 inhibitors OR sodium-glucose cotransporter 2 inhibitor OR SGLT-2i) AND (obesity OR corpulence OR fat OR overweight OR overload weight). We identified the included studies by browsing titles and abstracts, viewing full texts and supplementary materials.

\section{Study selection and quality assessment}

Inclusion criteria for this meta-analysis were as follows: (1) the overweight/obese, non-diabetic individuals were included in RCTs with SGLT-2i being used as monotherapy or as a supplement of non-surgical treatment in the intervention group; (2) vital parameters: changes in body weight, waist circumference, and metabolic indicators, including but not limited to fasting plasma glucose (FPG), blood pressure, lipids, and adverse effects were recorded in RCTs; (3) follow-up time $\geq 8$ weeks. Exclusion criteria: (1) nonEnglish language; (2) data unavailable; (3) duplicate reports. When the article was updated, the most recent or most complete article was selected.

Based on the above inclusion criteria, the two researchers independently evaluated the eligible studies, further discussed the parts causing disagreement, or asked a third researcher to review the controversial articles. The Cochrane Collaboration's tool was applied to accomplish quality assessment of the included studies and the risk of bias by reviewing and judging in terms of sequence generation, allocation concealment, blinding, incomplete outcome data, selective outcome reporting, and lack of other bias.

\section{Data extraction}

Data extracted from the studies included the following: (1) basic study characteristics, such as author, publication year, region, Clinical Trials.gov identifier, inclusion criteria, follow-up time, drugs and dose, or other interventions in the experimental group and control group; (2) outcomes, including weight-related changes; waist circumference changes; and metabolism-related indicators, such as FPG, blood pressure, lipids, etc.; (3) detailed data on adverse events occurred in the experimental and control groups. The data not available in the papers, including full texts and supplementary materials, will be supplemented by excerpting study results in Clinical Trials. gov (if it is registered). Data extraction was done independently by two researchers and reviewed by a third researcher.

\section{Statistical analysis}

Review Manager 5.3 statistical analysis software was used for this analysis. The weighted mean difference (WMD) or standardized mean difference (SMD) and 95\% confidence interval (CI) were calculated to assess continuous variables. Risk ratio (RR) and 95\% CI were calculated to evaluate dichotomous variables. Due to the different types of SGLT-2i included in the study and the diversity of intervention measures in the control group, we applied a random effects model for analysis. $p \leq 0.05$ was statistically significant. Publication bias was appraised via a funnel plot.

\section{Results}

\section{Search results and study characteristics}

A total of 3271 papers were identified: 3270 papers were searched from the database through the retrieval formula, and 1 paper was identified by other sources. 796 papers were left after deletion of duplicates. 771 articles were successively deleted by reviewing the titles and abstracts. After revision of 25 full texts, 12 were excluded, including 1 without available data, 5 duplicate studies, 5 with a study population of diabetic patients, and 1 with a follow-up time that was less than 8 weeks. Finally, a total of 13 studies were included for this meta-analysis [9-21] (Fig. 1).

The included studies were published from 2014 to 2021 and the range of their follow-up time was 8-48 weeks. All the included participants were overweight/obese (baseline BMI $\geq 25 \mathrm{~kg} / \mathrm{m}^{2}$ ), non-diabetic individuals. The experimental group was given SGLT-2i as an intervention, including canagliflozin, licogliflozin, dapagliflozin, empagliflozin, remogliflozin, and sergliflozin. The interventions that were applied to the control group included placebo, metformin, phentermine, exercise, and no intervention. These RCTs were conducted in different countries: 5 in the United States, 1 in the United States and Puerto Rico, 2 in the United Kingdom, 1 in Denmark, 1 in Mexico, 1 in Iran, 1 in Japan, and 1 multi-country, multicentre study (Tab. 1).

\section{Risk of bias assessment}

Due to incomplete information, the risk of sequence generation in 5 studies was unclear, and the risk in 8 studies was low. In terms of allocation concealment, 


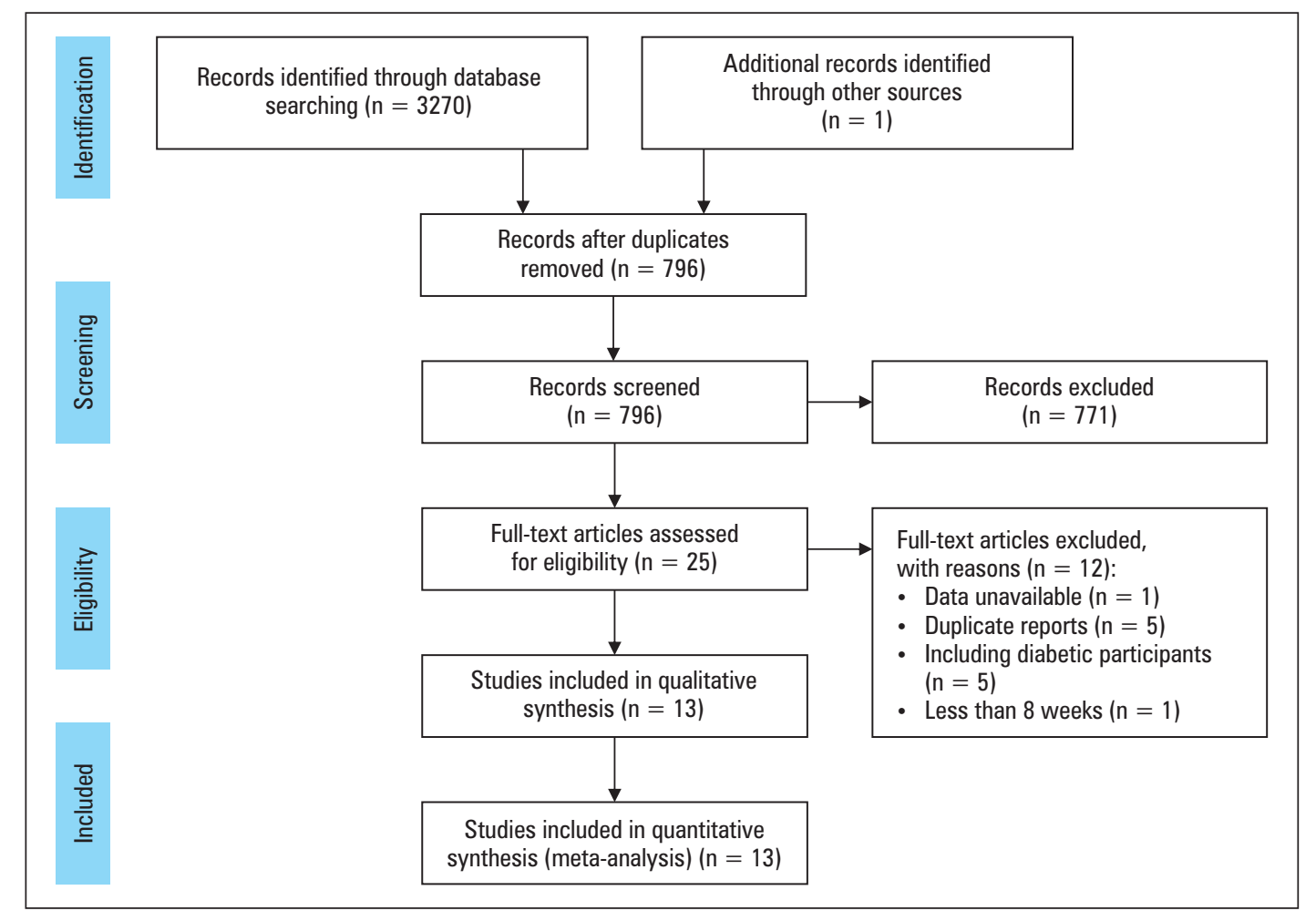

Figure 1. A schematic flow for selecting the articles included in this meta-analysis

7 studies were in low risk and 6 studies were unclear. As for blinding, 2 trials possessed high risk of bias, 1 was unclear, and the remaining 10 studies were low. In most studies, both incomplete outcome data and selective outcome reporting assessments were of low risk of bias, and only 1 study was unable to clearly determine the selective outcome reporting. In the aspect of lack of other bias, it was difficult to obtain accurate evaluations for all studies, some of which were thus judged as unclear (Supplementary File - Tab. 1).

\section{Efficacy outcomes on weight and waist circumference}

An analysis involving 648 participants showed that SGLT-2i significantly reduces body weight compared with the control group (WMD $=-1.33,95 \% \mathrm{CI}=-2.17$ to $-0.50, \mathrm{p}=0.002$ ) (Fig. 2A). Also, 3 studies indicated that SGLT-2i significantly reduces weight percentage $(\mathrm{WMD}=-1.52,95 \% \mathrm{CI}=-2.04$ to $-1.00, \mathrm{p}<0.001)$ (Fig. 2b).

Two studies with 540 participants involved revealed that SGLT-2i reduces waist circumference in overweight/obese, non-diabetic individuals (WMD $=-1.94$, $95 \% \mathrm{CI}=-3.73$ to $-0.14, \mathrm{p}=0.03$ ) (Fig. 3A). In addition, the percentage reduction in waist circumference in 103 subjects also indicated significant benefit of using SGLT-2i (WMD $=-3.00,95 \% \mathrm{CI}=-5.48$ to -0.52 , $\mathrm{p}=0.02)$ (Fig. 3B).
Furthermore, 345 of the 1645 participants in 5 studies experienced a $\geq 5 \%$ weight decrease. 208 out of the 743 participants in the SGLT-2i intervention group, and 137 of the 902 patients in the control group had weight loss $\geq 5 \%$. In short, SGLT-2i is significantly advantageous in weight decrease $(\geq 5 \%)(\mathrm{RR}=2.04,95 \% \mathrm{CI}=1.51$ to $2.76, \mathrm{p}<0.001$ ) (Fig. 4). Whereas, when weight loss was $\geq 10 \%$, there was no significant difference between SGLT-2i and the control group $(\mathrm{RR}=1.83,95 \% \mathrm{CI}=0.70$ to $4.76, \mathrm{p}=0.22$ ) when looking at the combined data of 12 weeks and 26 weeks from these 2 studies (Fig. 5).

\section{Other metabolic conditions}

In terms of blood glucose, data of 5 studies showed no significant effect of SGLT-2i on FPG (SMD $=-0.33$, $\mathrm{p}=0.27)$ in overweight/obese, non-diabetic individuals. Similarly, for blood pressure we found that, comparing with the controlled group, SGLT-2i was not correlated with systolic blood pressure (SBP) $(\mathrm{WMD}=1.86, \mathrm{p}=0.39)$ or diastolic blood pressure (DBP) (WMD =0.13, $\mathrm{p}=0.92)$. As for lipid-wise parameters, SGLT-2i was shown to have no significant effect on high-density lipoprotein cholesterol (HDL-C) $(\mathrm{SMD}=0.03, \mathrm{p}=0.86)$, low-density lipoprotein cholesterol $(\mathrm{LDL}-\mathrm{C})(\mathrm{SMD}=-0.07, \mathrm{p}=0.73)$, total cholesterol (TC) $(\mathrm{SMD}=-0.03, \mathrm{p}=0.87)$, and triglyceride $(\mathrm{TG})$ $(\mathrm{SMD}=-0.07, \mathrm{p}=0.69)$. We also found that alanine aminotransferase (ALT) $(\mathrm{WMD}=1.86, \mathrm{p}=0.39)$, aspar- 


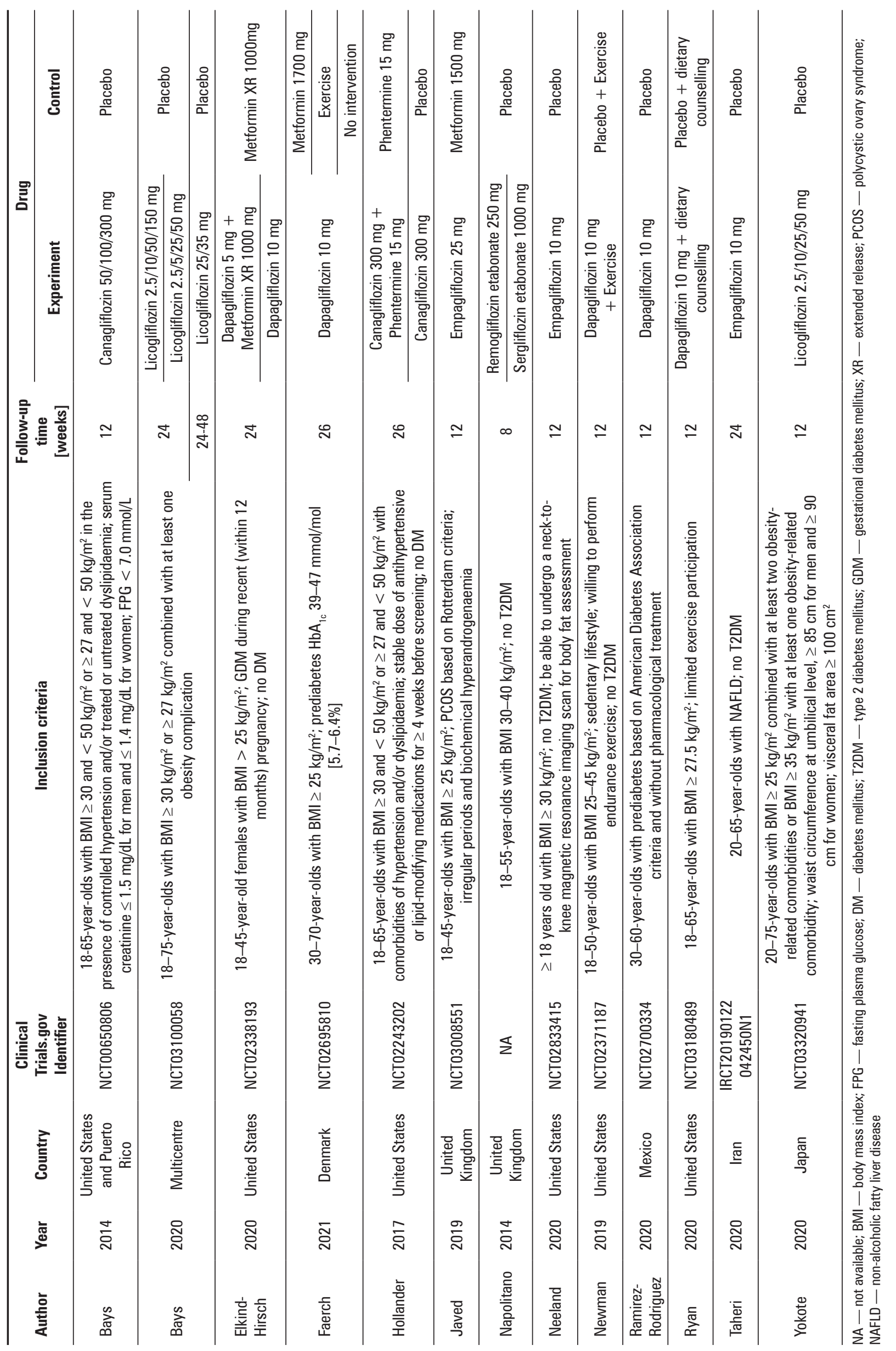




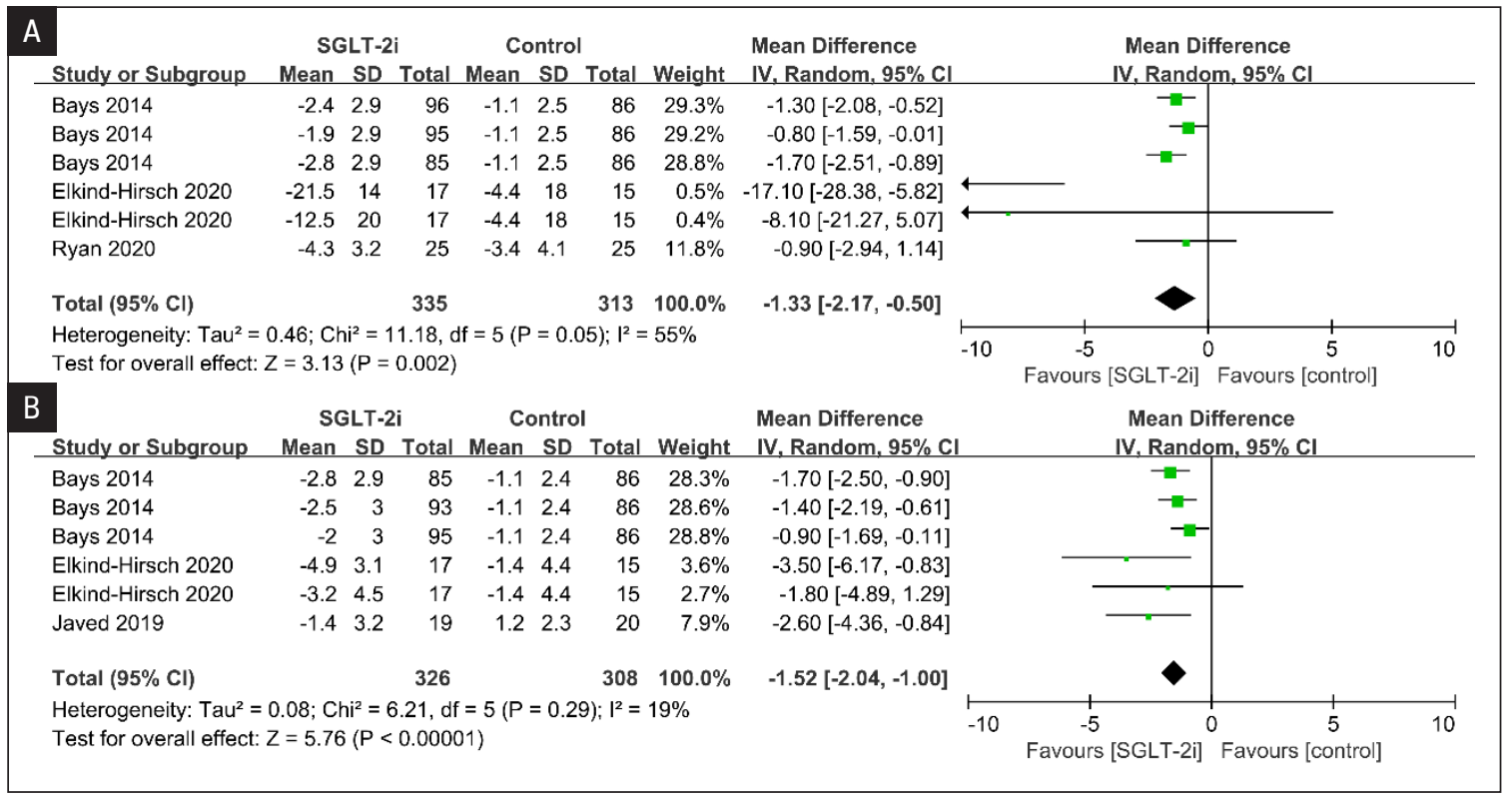

Figure 2. Effect on body weight of SGLT-2i compared to controls: weight change ( $\mathrm{kg})(\boldsymbol{A})$ and weight change (\%) (B)

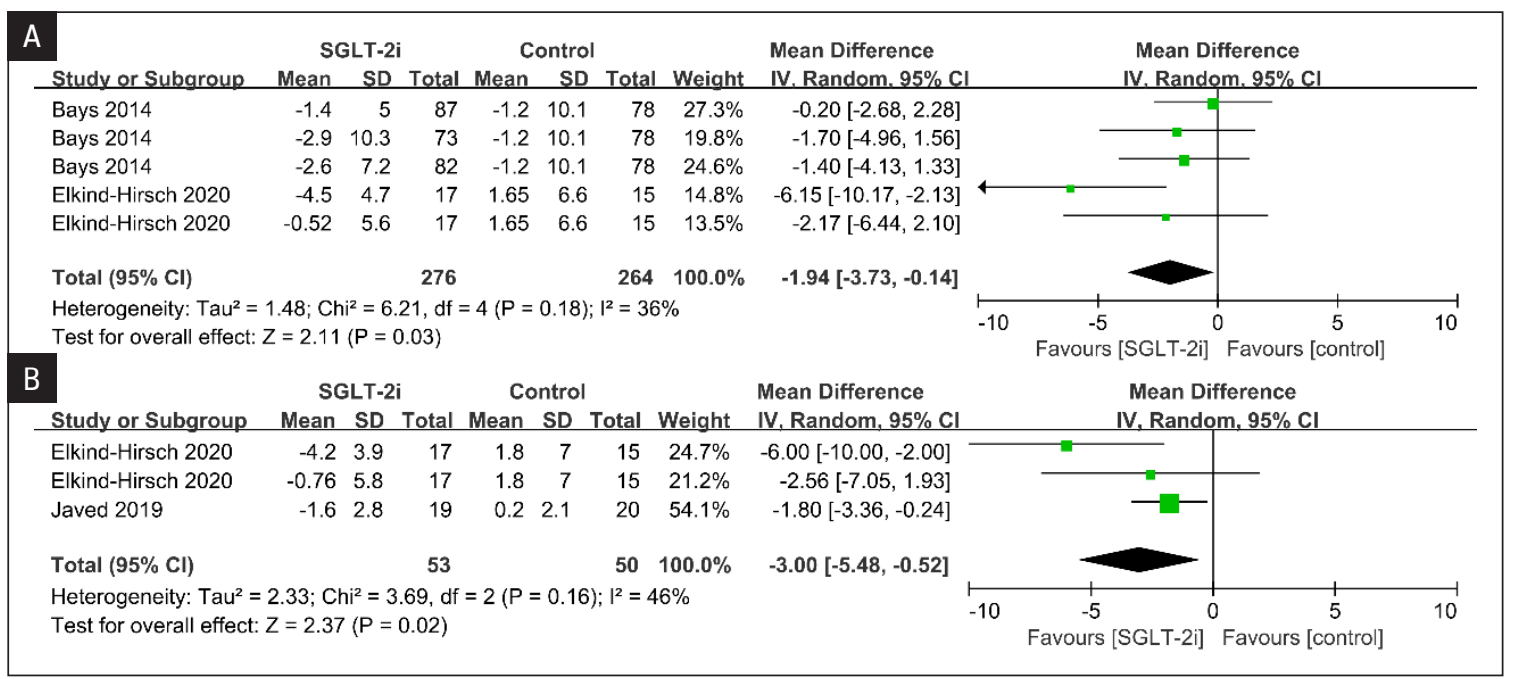

Figure 3. Effect on waist circumference of SGLT-2i compared to controls: waist circumference change (cm) (A), waist circumference change $(\%)(B)$

tate aminotransferase (AST) $(\mathrm{WMD}=1.84, \mathrm{p}=0.29)$, and homeostasis model assessment-insulin resistance (HOMA-IR) (WMD =0.12, $\mathrm{p}=0.58$ ) showed no difference between groups with and without intervention involving SGLT-2i (Tab. 2).

\section{Safety outcomes}

Analysis of any adverse events results of 8 studies, with 1671 participants involved, showed that SGLT-2i did not significantly increase the risk of total adverse events $(R R=1.09,95 \% \mathrm{CI}=0.99$ to $1.21, \mathrm{p}=0.08)$. However, it is worth noting that SGLT-2i has a risk of inducing infection. After analysing data from 6 RCTs, we reached a conclusion that SGLT-2i would increase the incidence of urinary tract infection $(R R=1.91$, $95 \% \mathrm{CI}=1.17$ to $3.11, \mathrm{p}=0.009)$, and 4 studies with 1327 participants demonstrated that SGLT-2i significantly raised the infectious risk in the reproductive system $(\mathrm{RR}=4.09,95 \% \mathrm{CI}=2.31$ to $7.26, \mathrm{p}<0.001)$. In addition, an analysis of 4 studies with 1208 participants illustrated that SGLT-2i potentially played a role in relieving diarrhoea $(\mathrm{RR}=0.48,95 \% \mathrm{CI}=0.26$ to $0.88, \mathrm{p}=0.02)$.

Analysis of other safety outcomes showed no significant effects of SGLT-2i in overweight/obese, non-diabetic individuals, including nausea, constipation, dizziness, 


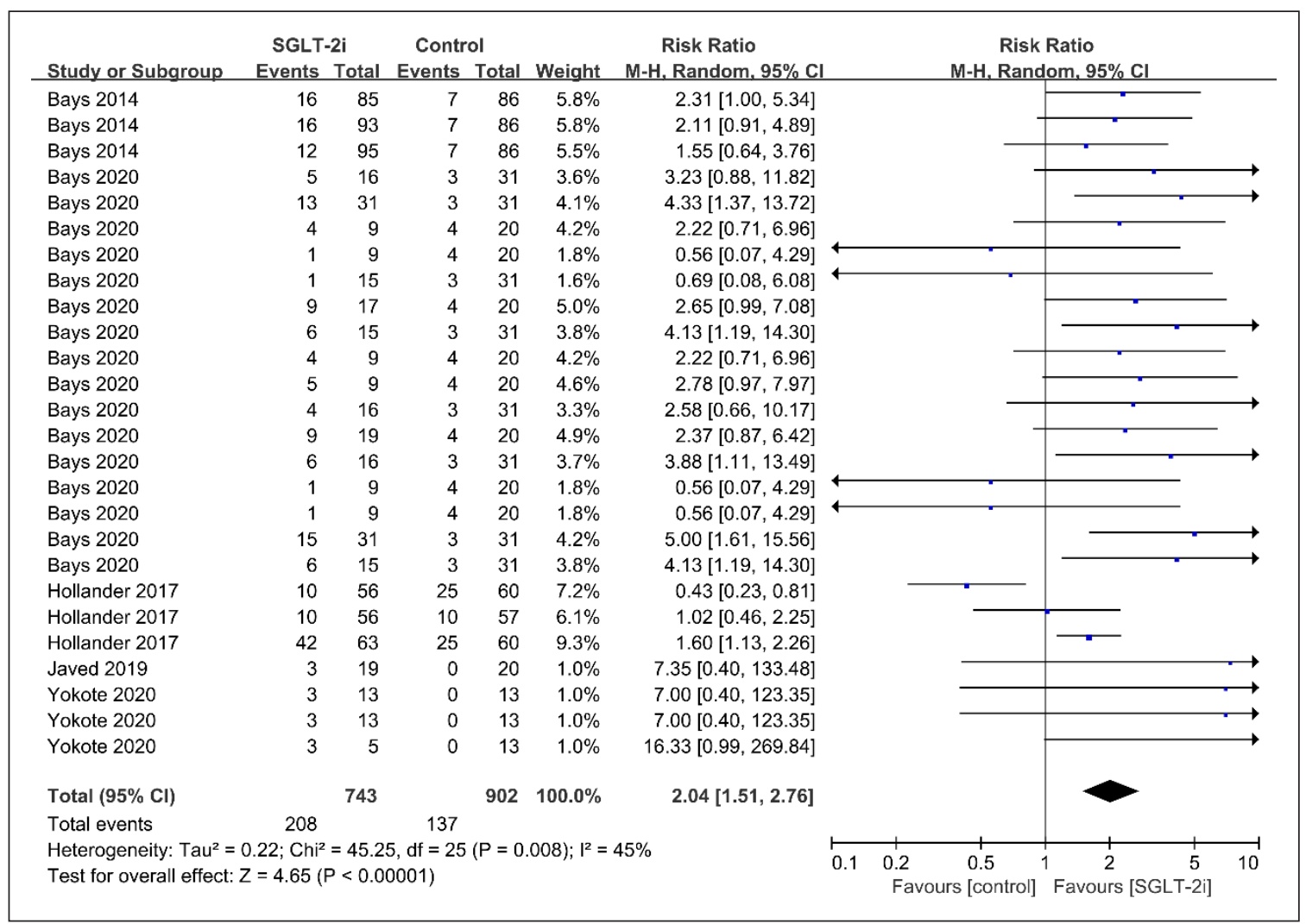

Figure 4. A forest plot of SGLT-2i compared to controls regarding weight decrease $\geq 5 \%$

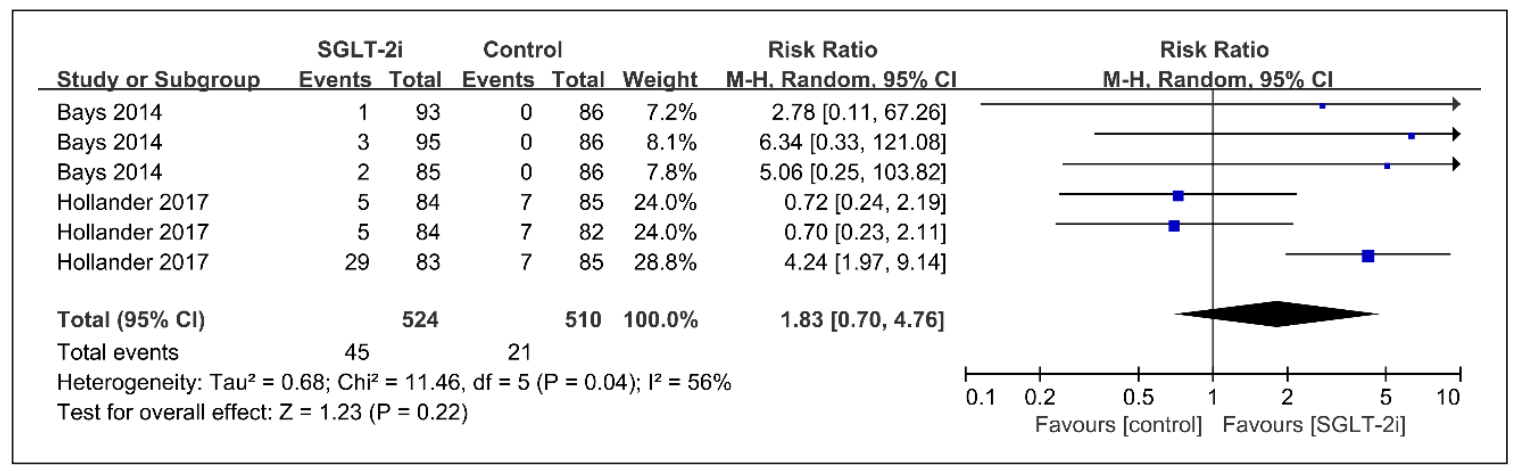

Figure 5. A forest plot of SGLT-2i compared to controls regarding weight decrease $\geq 10 \%$

headache, arthralgia, and allergy, compared with the control group (Tab. 3).

\section{Publication bias}

The results of publication bias test for the included studies gave a roughly symmetric funnel plot. There was no significant publication bias in this study.

\section{Discussion}

In a previous meta-analysis, SGLT-2i, used as an anti-hyperglycaemic agent, was investigated regarding its association with weight loss in T2DM patients com- pared with placebo [7]. Our meta-analysis evaluated the efficacy and safety of SGLT-2i in the overweight/obese with non-diabetic individuals by reviewing 13 RCTs with a follow-up duration of $\geq 8$ weeks.

According to the results of our study, SGLT-2i is potentially more effective than the control group in terms of reducing waist circumference and body weight loss $\geq 5 \%$ in overweight/obese, non-diabetic individuals. A retrospective cohort study showed that adding SGLT-2i in the metformin treatment for T2DM patients is significantly advantageous in weight loss $\geq 3 \%$, the number of people accounted for $53.6 \%$ (29.7\% for dipeptidyl-peptidase- 4 inhibitor [DPP-4i], $18.6 \%$ for 
Table 2. The difference in efficacy between sodium-glucose cotransporter 2 inhibitor (SGLT-2i) and control

\begin{tabular}{|c|c|c|c|c|c|c|c|}
\hline SGLT-2i vs. control & $\begin{array}{c}\text { No. } \\
\text { of studies }\end{array}$ & Participants & Effect size & Difference & $95 \% \mathrm{Cl}$ & $\mathbf{p}$ & $\begin{array}{l}\text { Heterogeneity } \\
\left(I^{2}\right)(\%)\end{array}$ \\
\hline FPG & 5 & 258 & SMD & -0.33 & $-0.93-0.26$ & 0.27 & 80 \\
\hline \multicolumn{8}{|l|}{ Blood pressure } \\
\hline SBP & 4 & 177 & WMD & 1.86 & $-2.40-6.12$ & 0.39 & 34 \\
\hline DBP & 3 & 138 & WMD & 0.13 & $-2.57-2.84$ & 0.92 & 0 \\
\hline \multicolumn{8}{|l|}{ Lipid } \\
\hline HDL-C & 4 & 177 & SMD & 0.03 & $-0.27-0.32$ & 0.86 & 0 \\
\hline LDL-C & 3 & 138 & SMD & -0.07 & $-0.47-0.33$ & 0.73 & 27 \\
\hline TC & 4 & 177 & SMD & -0.03 & $-0.36-0.30$ & 0.87 & 18 \\
\hline TG & 3 & 138 & SMD & -0.07 & $-0.40-0.27$ & 0.69 & 0 \\
\hline \multicolumn{8}{|l|}{ Aminotransferase } \\
\hline ALT & 3 & 178 & WMD & 1.86 & $-2.42-6.15$ & 0.39 & 0 \\
\hline AST & 3 & 178 & WMD & 1.84 & $-1.57-5.26$ & 0.29 & 29 \\
\hline HOMA-IR & 3 & 204 & WMD & 0.12 & $-0.31-0.54$ & 0.58 & 57 \\
\hline
\end{tabular}

$\mathrm{CI}$ — confidence interval; WMD — weighted mean difference; SMD — standardized mean difference; FPG — fasting plasma glucose; SBP — systolic blood pressure; DBP — diastolic blood pressure; HDL-C — high-density lipoprotein cholesterol; LDL-C — low-density lipoprotein cholesterol; TC — total cholesterol; TG — triglyceride; ALT — alanine aminotransferase; AST — aspartate aminotransferase; HOMA-IR — homeostasis model assessment-insulin resistance

Table 3. The incidence of adverse events between sodium-glucose cotransporter 2 inhibitor (SGLT-2i) and control

\begin{tabular}{lcccccc}
\hline SGLT-2i vs. control & $\begin{array}{c}\text { No. } \\
\text { of studies }\end{array}$ & Participants & RR & $\mathbf{9 5 \%} \mathbf{C l}$ & $\mathbf{p}$ & $\begin{array}{c}\text { Heterogeneity } \\
\left(\mathbf{I}^{\mathbf{2}}\right)(\%)\end{array}$ \\
\hline Any adverse events & 8 & 1671 & 1.09 & $0.99-1.21$ & 0.08 & 30 \\
\hline Nausea & 3 & 1117 & 1.41 & $0.63-3.14$ & 0.40 & 38 \\
\hline Diarrhoea & 4 & 1208 & 0.48 & $0.26-0.88$ & 0.02 & 0 \\
\hline Constipation & 3 & 1417 & 0.86 & $0.49-1.52$ & 0.60 & 1 \\
\hline Dizziness & 4 & 1261 & 2.01 & $0.98-4.13$ & 0.06 & 0 \\
\hline Headache & 4 & 1119 & 0.97 & $0.65-1.46$ & 0.90 & 0 \\
\hline Urinary tract infection & 6 & 1392 & 1.91 & $1.17-3.11$ & 0.009 & 0 \\
\hline Reproductive system infection & 4 & 1327 & 4.09 & $2.31-7.26$ & $<0.001$ & 0 \\
\hline Arthralgia & 2 & 743 & 0.93 & $0.33-2.58$ & 0.89 & 0 \\
\hline Allergies & 3 & 655 & 1.03 & $0.35-3.03$ & 0.96 & 0 \\
\hline
\end{tabular}

$\mathrm{RR}$ — risk ratio; $\mathrm{Cl}$ — confidence interval

sulfonylurea), and weight change from baseline $-3.47 \mathrm{~kg}$ (-1.21 kg for DPP-4i, $-0.04 \mathrm{~kg}$ for sulfonylurea) [22]. The 52-week DURATION-8 RCT showed weight loss $\geq 5 \%$ in the dapagliflozin group $(21.3 \%)$, which is more favourable than that in the exenatide group (14.1\%) in patients with T2DM poorly controlled with metformin treatment [23]. It is now believed that SGLT-2i can reduce the threshold of glucose excretion in renal urine by inhibiting the main glucose transporter in renal proximal tubules, resulting in glucose discharging in urine to reduce blood glucose [7,24]. This process leads further to partial heat loss and weight loss to a certain extent. The early stage of weight reduction may be caused by fluid loss, which is induced by mild osmotic diuresis. This influence diminishes with time, so in the later stage, the loss in weight is mainly caused by loss in fat mass [25].

Noticeably, the results of this study illustrated that the beneficial weight loss may be limited to $5 \%$ to $10 \%$. It is speculated that this range in weight loss may be due to the small sample size or the fact that the drugs themselves have limitations on weight loss in the overweight/obese, non-diabetic individuals. There are also studies suggesting that the actual weight loss is less than the expected weight loss predicted by the metabolic data, which may be due to the calorie loss caused by sugar via urine, resulting in compensatory overeating, but this has not been confirmed at present [26]. It is 
thought that as for T2DM patients, a more stringent diet management in addition to giving SGLT-2i may achieve greater control in blood glucose level and weight [27]. On the other hand, because sodium-glucose cotransporter 2 (SGLT2) is inhibited, sodium-glucose cotransporter 1 (SGLT1) may up-regulate reabsorption and partially inhibit renal glucose excretion, thus limiting the weight loss effect of SGLT-2i to a certain extent [28]. Therefore, some researchers proposed SGLT1/2 inhibitors, such as licogliflozin or sotagliflozin, which may be able to inhibit both SGLT1 and SGLT2, would play a more drastic role in weight loss than SGLT-2i [28]. Thus it can be recognized that the causes of weight loss in SGLT-2i therapy are multifaceted and may be more complex than we have illustrated at present [7].

Because SGLT-2i is a class of anti-hyperglycaemic agents that is used in diabetic treatments, the risk of hypoglycaemia should be taken into consideration when used in non-diabetic people. The results of this meta-analysis showed that SGLT-2i had no effect on the risk of hypoglycaemia in the overweight/obese, non-diabetic population, which is consistent with data recorded by the US Food and Drug Administration.

In terms of other metabolic indicators, SGLT-2i showed no significant effect on blood pressure, lipids, or aminotransferase in overweight/obese, non-diabetic individuals. An analysis of studies observed moderate reductions in SBP and DBP in treatments with SGLT-2i, which provides additional clinical benefit in the aspect of the cardiovascular system for the majority of T2DM patients [29]. Also, it has been reported that SGLT-2i may reduce plasma volume and blood pressure by promoting osmotic diuretic and sustained natriuretic effects in T2DM patients [30]. Improved ventricular load may be partly associated with cardiovascular protection [31]. Notably, Zannad et al. performed a DAPA-HF and EMPEROR-reduced meta-analysis showing that SGLT-2i (empagliflozin or dapagliflozin) reduces all-cause and cardiovascular death and hospitalisations for heart failure, and improves renal outcomes in patients with heart failure and a reduced ejection fraction with or without diabetes [32]. Li et al. found that SGLT-2i did not increase the risk of dyslipidaemia in T2DM patients overall, but the increase in BMI may elevate the risk [33]. A meta-analysis showed that SGLT-2i reduces alanine aminotransferase (ALT), and aspartate transaminase (AST) in patients with T2DM and protects the liver [34]. However, the trial of overweight patients with non-alcoholic fatty liver disease and without T2DM revealed that SGLT-2i does not change hepatic triacylglycerol content and does not give statistically significant improvement in hepatic steatosis after 12 weeks of administration [35].
There are some differences in results between our metabolic analysis and the above studies, which may be due to the small sample size included or the different internal environments in different populations. Most of the above studies focused on patients with T2DM, while this study was conducted in overweight/obese individuals without diabetic conditions. Current studies fail to focus on the obese population without diabetes. This meta-analysis paves a path to a promising future of applying SGLT-2i for anti-obesity treatment.

As for safety, SGLT-2i administration was generally well tolerated in the overweight/obese, non-diabetic individuals. It has been widely studied that SGLT-2i treatment is associated with increased risk of urinary tract and genital infections, which may be closely related to the mechanism of action of the drugs. Most of these infections are mild to moderate. SGLT-2i elevates glucose level in urine. The glucosuria environment can promote the growth and reproduction of bacteria, and the environment is also an inducer of increasing bacterial adhesion to the uroepithelium, resulting in increased risk of infections in the urinary tract and genitals [36]. In addition, participants in the SGLT-2i intervention groups had significantly fewer adverse events in terms of diarrhoea than those in the control groups. The increased diarrhoea appearance may be caused by the use of metformin in some participants in the control groups in this meta-analysis study. Studies have found that metformin is prone to cause adverse drug reactions in the gastrointestinal tract, most commonly diarrhoea and nausea [37]. In comparison, SGLT-2i appeared to reduce the risk of diarrhoea.

All data in this meta-analysis were from RCTs, and the quality of each study was assessed in the process of inclusion. However, the existence of limitations is undeniable. Firstly, due to the limited number of studies and inconsistent follow-up time, the extracted outcome indicators were not analysed separately according to the follow-up time, so it is difficult to judge whether the different duration of SGLT-2i use has any more specific and finer effects on treating overweight/obese, non-diabetic individuals. Moreover, this study did not address the longer-term efficacy and safety of SGLT-2i treatment (maximum follow-up time of 48 weeks in the included study). Finally, due to the variety in dosage, type, etc. of SGLT-2i and limited sample size of these RCTs, the relationships between dosage and weight loss and other outcomes were not included in this study. Although there are limitations, this study is the first meta-analysis that focus on the effects of SGLT-2i treatment in overweight/obese, non-diabetic individuals, which is a potentially new option for managing obesity. Future studies are essential in the aspects mentioned above. 


\section{Conclusion}

SGLT-2i is associated with a limited loss in weight and waist circumference in overweight/obese, non-diabetic individuals. There was no statistically significant effect on other metabolic parameters, including FPG, blood pressure, lipids, aminotransferase, and HOMA-IR. In terms of safety, SGLT-2i significantly increases the risk of urogenital infections. As for future prospects, more studies with higher quality, longer-term trials as well as larger samples should be carried out to investigate the efficacy and safety of using SGLT-2i in the management of overweight/obese, non-diabetic individuals.

\section{References}

1. Hruby A, Hu FB. The Epidemiology of Obesity: A Big Picture. Pharmacoeconomics. 2015; 33(7): 673-689, doi: 10.1007/s40273-014-0243-x, indexed in Pubmed: 25471927.

2. Hendryx M, Chojenta C, Byles JE. Obesity Risk Among Young Australian Women: A Prospective Latent Class Analysis. Obesity (Silve Spring). 2020; 28(1): 154-160, doi: 10.1002/oby.22646, indexed in Pubmed: 31755240 .

3. Kabiri M, Sexton Ward A, Ramasamy A, et al. The Societal Value of Broader Access to Antiobesity Medications. Obesity (Silver Spring).2020; 28(2): 429-436, doi: 10.1002/oby.22696, indexed in Pubmed: 31869002.

4. Cheng Ji, Gao J, Shuai X, et al. The comprehensive summary of surgical versus non-surgical treatment for obesity: a systematic review and meta-analysis of randomized controlled trials. Oncotarget. 2016; 7(26): 39216-39230, doi: 10.18632/oncotarget.9581, indexed in Pubmed: 27233078.

5. Tak YI, Lee SY. Long-Term Efficacy and Safety of Anti-Obesity Treatment: Where Do We Stand? Curr Obes Rep. 2021; 10(1): 14-30 doi: 10.1007/s13679-020-00422-w, indexed in Pubmed: 33410104.

6. Tilinca MC, Tiuca RA, Niculas $C$, et al. Future perspectives in diabesity treatment: Semaglutide, a glucagon-like peptide 1 receptor agonis (Review). Exp Ther Med. 2021; 22(4): 1167, doi: 10.3892/etm.2021.10601, indexed in Pubmed: 34504612 .

7. Cai X, Yang W, Gao X, et al. The Association Between the Dosage of SGLT2 Inhibitor and Weight Reduction in Type 2 Diabetes Patients: A Meta-Analysis. Obesity (Silver Spring). 2018; 26(1): 70-80, doi: 10.1002/oby.22066, indexed in Pubmed: 29165885.

8. Liberati A, Altman DG, Tetzlaff J, et al. The PRISMA statement for reporting systematic reviews and meta-analyses of studies that evaluate health care interventions: explanation and elaboration. Ann Intern Med. 2009; 151(4): W65-W94, doi: 10.7326/0003-4819-151-4-200908180-00136 indexed in Pubmed: 19622512.

9. Bays HE, Kozlovski P, Shao Q, et al. Licogliflozin, a Novel SGLT1 and 2 Inhibitor: Body Weight Effects in a Randomized Trial in Adults with Overweight or Obesity. Obesity (Silver Spring). 2020; 28(5): 870-881, doi: 10.1002/oby.22764, indexed in Pubmed: 32187881.

10. Bays HE, Weinstein R, Law G, et al. Canagliflozin: effects in overweight and obese subjects without diabetes mellitus. Obesity (Silver Spring). 2014; 22(4): 1042-1049, doi: 10.1002/oby.20663, indexed in Pubmed: 24227660.

11. Elkind-Hirsch KE, Seidemann E, Harris R. A randomized trial of dapagliflozin and metformin, alone and combined, in overweight wome after gestational diabetes mellitus. Am J Obstet Gynecol MFM. 2020; 2(3): 100139, doi: 10.1016/j.ajogmf.2020.100139, indexed in Pubmed: 33345876

12. Færch K, Blond MB, Bruhn L, et al. The effects of dapagliflozin, metformin or exercise on glycaemic variability in overweight or obese individual with prediabetes (the PRE-D Trial): a multi-arm, randomised, controlled trial. Diabetologia. 2021; 64(1): 42-55, doi: 10.1007/s00125-020-05306-1, indexed in Pubmed: 33064182.

13. Hollander P, Bays HE, Rosenstock J, et al. Coadministration of Canagliflozin and Phentermine for Weight Management in Overweigh and Obese Individuals Without Diabetes: A Randomized Clinical Trial. Diabetes Care. 2017; 40(5): 632-639, doi: 10.2337/dc16-2427, indexed in Pubmed: 28289041.

14. Javed Z, Papageorgiou M, Deshmukh H, et al. Effects of empagliflozin on metabolic parameters in polycystic ovary syndrome: A randomized controlled study. Clin Endocrinol (Oxf). 2019; 90(6): 805-813, doi: 10.1111/cen.13968, indexed in Pubmed: 30866088.

15. Napolitano A, Miller S, Murgatroyd PR, et al. Exploring glycosuria as a mechanism for weight and fat mass reduction. A pilot study with remogliflozin etabonate and sergliflozin etabonate in healthy obese subjects. J Clin Transl Endocrinol. 2014; 1(1): e3-e8, doi: 10.1016/j. jcte.2013.12.001, indexed in Pubmed: 29235586.

16. Neeland IJ, de Albuquerque Rocha N, Hughes C, et al. Effects of Empagliflozin Treatment on Glycerol-Derived Hepatic Gluconeogenesis in Adults with Obesity: A Randomized Clinical Trial. Obesity (Silver Spring). 2020; 28(7): 1254-1262, doi: 10.1002/oby.22854, indexed in Pubmed: 32568464

17. Newman AA, Grimm NC, Wilburn JR, et al. Influence of Sodium Glucose Cotransporter 2 Inhibition on Physiological Adaptation to Endurance Exercise Training. J Clin Endocrinol Metab. 2019; 104(6): 1953-1966, doi: 10.1210/jc.2018-01741, indexed in Pubmed: 30597042.

18. Ramírez-Rodríguez AM, González-Ortiz M, Martínez-Abundis E. Effect of Dapagliflozin on Insulin Secretion and Insulin Sensitivity in Patients with Prediabetes. Exp Clin Endocrinol Diabetes. 2020; 128(8): 506-511, doi: 10.1055/a-0664-7583, indexed in Pubmed: 30149417.

19. Ryan SPP, Newman AA, Wilburn JR, et al. Sodium Glucose Co-Transporter 2 Inhibition Does Not Favorably Modify the Physiological Responses to Dietary Counselling in Diabetes-Free, Sedentary Overweight and Obese Adult Humans. Nutrients. 2020; 12(2), doi: 10.3390/nu12020510, indexed in Pubmed: 32085394

20. Taheri H, Malek M, Ismail-Beigi F, et al. Effect of Empagliflozin on Liver Steatosis and Fibrosis in Patients With Non-Alcoholic Fatty Liver Disease Without Diabetes: A Randomized, Double-Blind, Placebo-Controlled Trial. Adv Ther. 2020; 37(11): 4697-4708, doi: 10.1007/s12325-020-01498-5, indexed in Pubmed: 32975679.

21. Yokote K, Sano M, Tsumiyama I, et al. Dose-dependent reduction in body weight with LIK066 (licogliflozin) treatment in Japanese patients with obesity. Diabetes Obes Metab. 2020; 22(7): 1102-1110, doi: 10.1111/dom.14006, indexed in Pubmed: 32072763.

22. Vlacho B, Mundet-Tudurí X, Mata-Cases M, et al. Analysis of the effectiveness of second oral glucose-lowering therapy in routine clinica practice from the mediterranean area: A retrospective cohort study. Diabetes Res Clin Pract. 2021; 171: 108616, doi: 10.1016/j.diabres.2020.108616, indexed in Pubmed: 33310172.

23. Jabbour SA, Frías JP, Ahmed A, et al. Exenatide once weekly plus dapagliflozin once daily versus exenatide or dapagliflozin alone in patients with type 2 diabetes inadequately controlled with metformin monotherapy (DURATION-8): a 28 week, multicentre, double-blind, phase 3, randomised controlled trial. Lancet Diabetes Endocrinol. 2016; 4(12): 1004-1016, doi: 10.1016/S2213-8587(16)30267-4, indexed in Pubmed: 27651331.

24. Heerspink HJL, Kosiborod M, Inzucchi SE, et al. Renoprotective effects of sodium-glucose cotransporter-2 inhibitors. Kidney Int. 2018; 94(1): 26-39, doi: 10.1016/j.kint.2017.12.027, indexed in Pubmed: 29735306.

25. Sha S, Polidori D, Heise T, et al. Effect of the sodium glucose co-transporter 2 inhibitor canagliflozin on plasma volume in patients with type 2 diabetes mellitus. Diabetes Obes Metab. 2014; 16(11): 1087-1095, doi: 10.1111/dom.12322, indexed in Pubmed: 24939043.

26. Rajeev SP, Cuthbertson DJ, Wilding JPH. Energy balance and metabolic changes with sodium-glucose co-transporter 2 inhibition. Diabetes Obes Metab. 2016; 18(2): 125-134, doi: 10.1111/dom.12578, indexed in Pubmed: 26403227.

27. Ferrannini G, Hach T, Crowe S, et al. Energy Balance After Sodium-Glucose Cotransporter 2 Inhibition. Diabetes Care. 2015; 38(9): 1730-1735, doi: 10.2337/dc15-0355, indexed in Pubmed: 26180105.

28. Rebello CJ, Greenway FL. Obesity medications in development. Expert Opin Investig Drugs. 2020; 29(1): 63-71, doi: 10.1080/13543784.2020.170 5277, indexed in Pubmed: 31847611.

29. Reed JW. Impact of sodium-glucose cotransporter 2 inhibitors on blood pressure. Vasc Health Risk Manag. 2016; 12: 393-405, doi: 10.2147/VHRM. S111991, indexed in Pubmed: 27822054.

30. Mazidi M, Rezaie P, Gao HK, et al. Effect of Sodium-Glucose Cotransport-2 Inhibitors on Blood Pressure in People With Type 2 Diabetes Mellitus: A Systematic Review and Meta-Analysis of 43 Randomized Control Trials With 22528 Patients. J Am Heart Assoc. 2017; 6(6), doi: 10.1161/JAHA.116.004007, indexed in Pubmed: 28546454.

31. Verma S, McMurray JJV. SGLT2 inhibitors and mechanisms of cardiovascular benefit: a state-of-the-art review. Diabetologia. 2018; 61(10): 2108-2117, doi: 10.1007/s00125-018-4670-7, indexed in Pubmed: 30132036.

32. Packer M, Butler J, Filippatos G, et al. EMPEROR Trial Committees and Investigators. SGLT2 inhibitors in patients with heart failure with reduced ejection fraction: a meta-analysis of the EMPER OR-Reduced and DAPA-HF trials. Lancet. 2020; 396(10254): 819-829, doi: 10.1016/S0140-6736(20)31824-9, indexed in Pubmed: 32877652.

33. Li D, Wu T, Wang T, et al. Effects of sodium glucose cotransporter 2 inhibitors on risk of dyslipidemia among patients with type 2 diabetes: A systematic review and meta-analysis of randomized controlled trials. Pharmacoepidemiol Drug Saf. 2020; 29(5): 582-590, doi: 10.1002/pds.4985, indexed in Pubmed: 32124527.

34. Simental-Mendía M, Sánchez-García A, Rodríguez-Ramírez M, et al. Effect of sodium-glucose co-transporter 2 inhibitors on hepatic param- 
eters: A systematic review and meta-analysis of randomized controlled trials. Pharmacol Res. 2021; 163: 105319, doi: 10.1016/j.phrs.2020.105319, indexed in Pubmed: 33246172

35. Marjot T, Green CJ, Charlton CA, et al. Sodium-glucose cotransporter 2 inhibition does not reduce hepatic steatosis in overweight, insulin-resistant patients without type 2 diabetes. JGH Open. 2020; 4(3): 433-440, doi: 10.1002/jgh3.12274, indexed in Pubmed: 32514450.
36. Geerlings S, Fonseca V, Castro-Diaz D, et al. Genital and urinary tract infections in diabetes: impact of pharmacologically-induced glucosuria. Diabetes Res Clin Pract. 2014; 103(3): 373-381, doi: 10.1016/j. diabres.2013.12.052, indexed in Pubmed: 24529566.

37. Bonnet F, Scheen A. Understanding and overcoming metformin gastrointestinal intolerance. Diabetes Obes Metab. 2017; 19(4): 473-481, doi: 10.1111/dom.12854, indexed in Pubmed: 27987248. 